\title{
Simulation Tools for Interpretation of High Resolution SAR Images of Urban Areas
}

\author{
Giorgio Franceschetti, Raffaella Guida, \\ Antonio Iodice, Daniele Riccio, Giuseppe Ruello \\ DIET \\ Università Federico II \\ Napoli, Italy \\ \{gfrance, rafguida, iodice, daniele.riccio, ruello\}@unina.it
}

\author{
Uwe Stilla \\ Photogrammetry \& Remote Sensing \\ Technische Universität \\ München, Germany \\ stilla@tum.de
}

\begin{abstract}
New powerful spaceborne sensors for monitoring urban areas have been designed and are ready for launch. More detailed SAR images will be soon available and, consequently, new tools for their interpretation are needed, above all when urban scenes are illuminated.

In this paper, the authors propose some tools for the study and the analysis of high resolution SAR images based on a SAR raw signal simulator for urban areas.

Comparing simulated SAR images with the real one, interpretation of SAR data is improved and fundamental support of the employed tools is further assessed.
\end{abstract}

\section{INTRODUCTION}

Nowadays, in every country urban areas are growing and expanding. For our safety, a constant monitoring of this expansion, as well as of health conditions of urban centers, is becoming an imperative necessity.

Fortunately, in the last decades new technologies have been developed to this aim. In the field of remote sensing, an important role is played by Synthetic Aperture Radars (SAR) working day and night in all weather conditions.

Because of poor spatial resolutions in the provided images, an Earth mapping by SAR has been mainly limited, in these years, to natural landscapes where the phenomena to be observed did not need high image resolution as they could be monitored on scales of many tens of meters.

But, unluckily, this is not true for urban areas. Here, in fact, the main structure of the objects in the scene (buildings, streets, etc..) has dimensions that are often in the scale of tens of meters while many other items are even smaller (windows, balconies, cars and so on). Consequently, high resolution is an important requirement for an adequate, not rough description of this kind of scene.

In the upcoming future, this condition will be satisfied by a new generation of SAR sensors. An example is given by the radar TerraSAR-X [1] which will be soon launched by the German Aerospace Center (DLR). With spatial resolutions in the range of 1 meter, TerraSAR-X will certainly improve urban areas monitoring and encourage the development of interesting applications. For example, great attention is given today to the procedures for retrieving geometrical information from SAR images of urban areas, [1]-[3]. Some efforts are also made in retrieving electromagnetic information even if, for now, only theoretic steps have been formulated [4].

In both cases, SAR images simulators are indispensable to test the feature extraction algorithms when the real images are not yet available.

In order to foresee the potential of high resolution on SAR images of urban areas, some experiments have been jointly carried out by the remote sensing groups at the University of Naples (Italy) and the University of Munich (Germany) and they are presented in this paper.

In Section 2 typical effects of SAR urban images are discussed with particular reference to a SAR image relevant to an area in the center of Munich (Germany).

The image interpretation is supported by some simulation tools described and employed, for the case at issue, in Section 3. More precisely, different kinds of simulations considering both the geometric and electromagnetic properties of the scene have been carried out.

In Section 4, simulated SAR images are compared with the real one, obtained during a flight campaign, in order to attempt an interpretation of some effects present in the real image.

Finally, remarkable comments and conclusions are reported.

\section{SAR URBAN IMAGES}

Often, SAR images are really difficult to be interpreted: the presence of speckle as well as of some distortion effects, like shadowing and layover, makes the analysis of this kind of image complex.

This is in particular true for SAR images of urban areas. Here, in fact, not only the above distortions are accentuated for the presence of many buildings close to each other, but these structures let multiple scattering arise which, in turn, produces new effects on the SAR image.

An example of complexness of urban SAR image follows.

Fig. 1 shows an optical aerial view of the test area. It is located in the center of Munich (Germany) and presents the group of buildings of the Technische Universität (in the center) 
and the Alte Pinakothek (at the right bottom) in the quarter of Schwabing. In an area smaller than $(600 \mathrm{~m})^{2}$ we find a great variety of objects and materials: big buildings and little structures, apex and plane roofs, circular and flat walls, gardens and pavements, concrete or brick surfaces, etc..

In each case, interaction with the radar signal is different and, moreover, there is also an electromagnetic mutual interaction among these structures when the scene is hit by radiation.

All this affects the relevant SAR image given in Fig. 2. It is a byte scaled version of the original SAR image in X-band acquired during an airborne flight campaign in which the radar functioning mode parameters have been set to achieve spatial resolution of $2.0 \mathrm{~m}$ and $3.0 \mathrm{~m}$ respectively in ground range and azimuth. The radar flight trajectory is aligned with the bottom side of the image, as can be deduced from the shadowing areas.

The presence of many effects in the SAR images was expected. For example, when the radar flight trajectory and look angle are known we can imagine the shadow direction and extension.

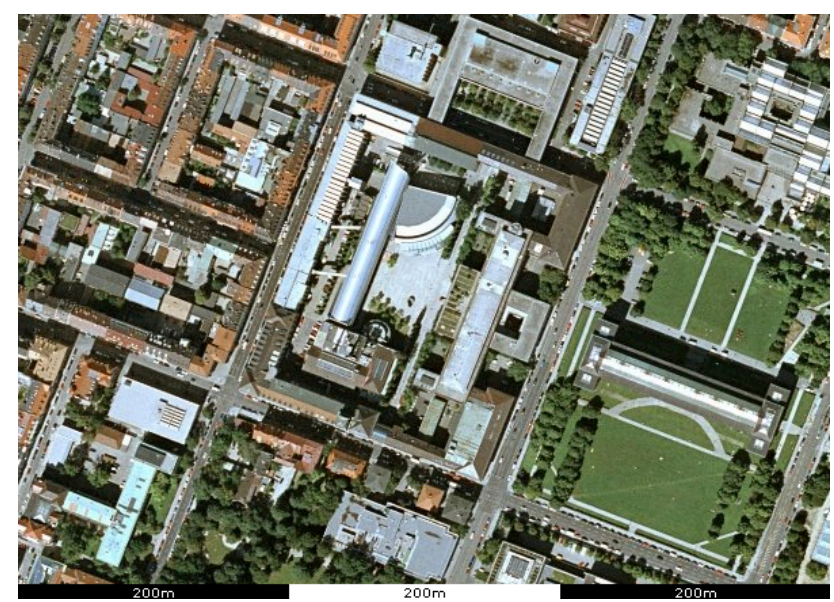

Figure 1. Airborne optical image of TUM and Alte Pinakothek. (GeoContent GmbH).

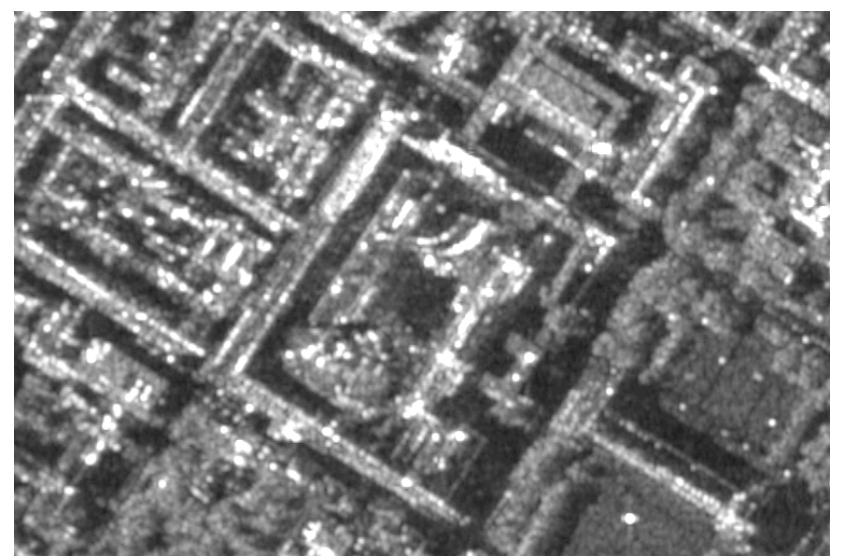

Figure 2. SAR image of the urban area in Fig. 1. The radar flight trajectory is aligned with the bottom side of the image. The image was taken with the E-SAR System (DLR-HR).
In a similar way, it is possible to foresee layover effects for all vertical surfaces, obviously numerous, in an urban scene.

But not everything can be predicted or interpreted by the only knowledge of SAR geometry. More precisely, an accurate description of the SAR image is not possible if our considerations are not based on a scattering model taking into account, at least for simple structures, both the geometric and electromagnetic parameters describing the scene. A proof of this statement is given in the following sections.

\section{Simulation TOOLS}

In this section we propose some tools for the study and the analysis of high resolution SAR images as a SAR raw signal simulator for urban areas [5]. It is an efficient simulator based on a frequency domain approach employing Fast Fourier Transforms (FFT) codes where a complete electromagnetic model of scattering from isolated buildings is implemented [6].

Different kinds of simulations considering both the geometric and electromagnetic properties of the scene have been carried out in order to show the great support can be provided by similar tools.

A first simulation has been realized to highlight the effects caused on the SAR image mainly by the geometric properties of the buildings in the scene. It means that no difference in materials has been considered, i.e. the same dielectric constant has been assumed for all the objects in the scene. Moreover, in this first step, we consider only contributions of the first order (i.e., single bounce) to the radar cross section.

To this aim, LIDAR data available on the test area have been adopted and the relevant surface profile is shown in Fig. 3 . In particular, an area of $400 \times 600 \mathrm{~m}^{2}$ is represented with 1 meter resolution in the axes directions and 0.2 meter resolution in the altitude profile. Technische Universität buildings and the Alte Pinakothek are clearly distinguishable.

The LIDAR data has been the input of the SAR raw signal simulator in which radar parameters have been set to obtain image resolutions of $0.27 \mathrm{~m}$ and $2.33 \mathrm{~m}$ respectively for the azimuth and the ground range. After processing the SAR raw signal, a multilook has been applied along the azimuth direction leading to a final resolution of $2.18 \mathrm{~m} \times 2.33 \mathrm{~m}$.

The resulting simulated SAR image is reported in the azimuth/slant range plane in Fig. 4.

Obviously, Fig. 4 shows only some effects expected for the studied area. In fact, accounting only for first order contributions to the radar cross section means that only single scattering is represented and, consequently, only layover and shadow areas are clearly visible. Instead, looking at the real SAR image in Fig. 2, we note many strips due to the double scattering that have not been represented in the simulation in Fig. 4.

It can be regarded as reductive but, in a first analysis, it is useful to retrieve information on geometrical properties of the buildings, above all when different views, i.e. different radar flight trajectories, are considered at the same time [3]. Anyway, simply observing Fig. 4, we are able to get a general idea of building dimensions and circular vertical surfaces can be distinguished from flat ones. 


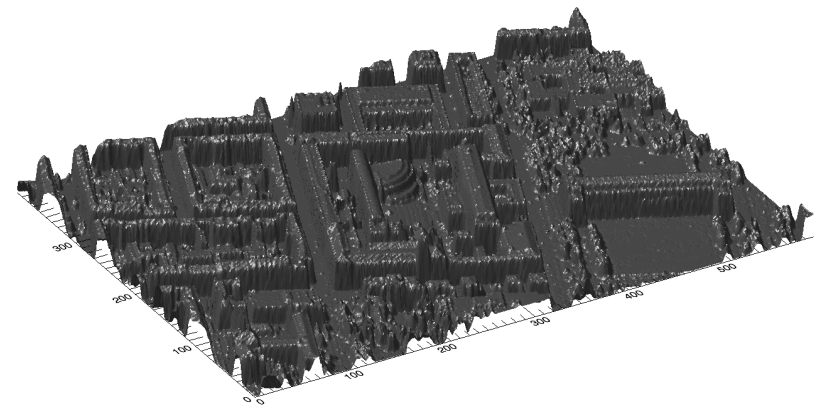

Figure 3. LIDAR data acquired on the urban area in Fig. 1 (axes are labeled in meter).

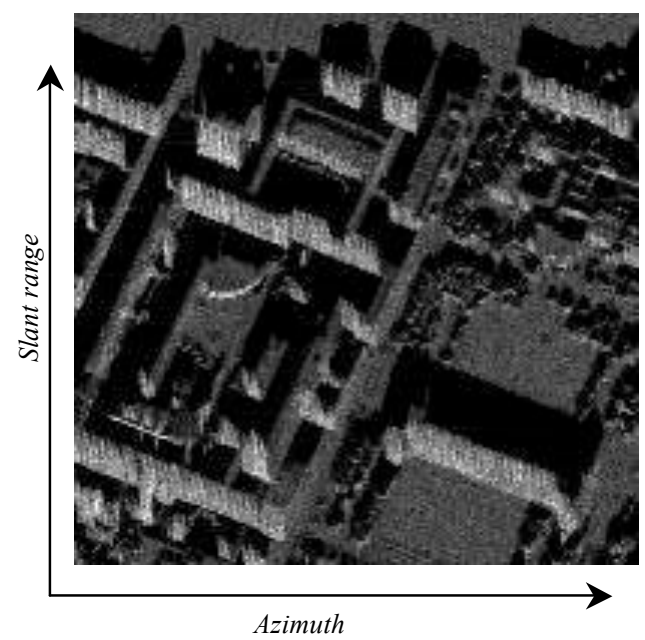

Figure 4. SAR simulated image relevant to the LIDAR data in Fig. 3

In order to consider also higher order contributions (i.e., multiple bounces), arising from dihedral and trihedral configurations formed by a building with the soil on which it is placed and the other structures nearby, we work in a different way.

Clearly, a simulation tool can not take into account all these contributions when efficiency and low computational costs are required. This leads us to assume a simplified geometrical model for the building (in practice an isolated parallelepiped on a rough terrain) in favour of a better representation of scattering mechanisms [6].

In this way, in spite of the geometry simplification, we can more satisfactorily explain some effects in the real SAR image.

In fact, we can now represent on the simulated SAR image also contributions of second and third order (higher order contributions do not arise for the geometric model adopted).

Indeed triple scattering, even if represented, is not clearly visible both in real and simulated images because it goes back to the radar too attenuated.

Double reflection, instead, is for us of great interest and its representation on simulated SAR images too is helpful for a better interpretation of real SAR images.
This contribution, in fact, brings an important information content which, if retrieved, can be used for supporting image analysis. When Kirchhoff approach with Geometric Optics is a good approximation for representing the signal backscattered to the sensor, the double reflection contribution to the radar cross section $\sigma^{o}$ is given by the expression below:

$$
\sigma^{\circ}=\frac{\left|S_{p q}\right|^{2} h l \tan \vartheta \cos \varphi\left(1+\tan ^{2} \vartheta \sin ^{2} \varphi\right)}{8 \pi^{2} \cos ^{2} \vartheta \cdot \sigma^{2}\left(2 / L^{2}\right) \cdot \exp \left[\frac{\tan ^{2} \vartheta \sin ^{2} \varphi}{2 \sigma^{2}\left(2 / L^{2}\right)}\right]}
$$

where $S_{p q}$ is the generic element of scattering matrix, with $p$ and $q$ each standing for $h$ or $v$ (horizontal or vertical polarization), $h$ and $l$ are building height and length, $\theta$ is the radar look angle, $\varphi$ is the wall orientation, $\sigma$ and $L$ are, respectively, deviation standard and correlation length of the stochastic process describing the soil roughness.

In $S_{p q}$ the electromagnetic behaviour of surfaces causing the double bounce of the signal, i.e. the building frontal wall and the rough soil, is considered through the dielectric constants of their own materials.

In this way, for double reflection as well as for single and triple scattering, we can represent the different answers of grass, bricks, concrete, asphalt etc..

The most important consequence of this approach is that not only what happens in the real world is better represented but it can be also better interpreted. Equation 1, in fact, asserts that, should the double reflection contribution be known, we could retrieve information about any geometric or electromagnetic parameter involved in this process, provided that a certain ground truth is given.

Even if all necessary information is not available, this kind of simulation tools can support human interpretation of SAR image as shown in the next Section.

\section{IMAGE INTERPRETATION}

Common experience says that in SAR images some effects are often unexpected.

An example is given also in Fig. 2. Let us look again at that image with more attention at the building of the Alte Pinakothek.

A certain ground truth is known about that area, in particular buildings dimensions are given. Such information relevant to the picture-gallery reveals that the structure is perfectly symmetric.

Moreover, the garden surrounding the building is symmetric respect to an axis perpendicular to the large frontal wall and passing through its center.

In these conditions, should we consider only geometric properties of the strucutures, we could never explain the effect appearing in the SAR image: a geometrically symmetric structure presents a not radiometrically symmetric strip of double reflection.

Looking inside Eq.(1) we can find an explanation of this effect. 
We know, by the ground truth, that the building is symmetric. Consequently, we expect that each section has the same height $h$.

The garden in front of the wall is mainly constituted by grass for which the same roughness, in a statistical sense, can be assumed for all its extension. This implies that the same couple of roughness parameters $(\sigma, L)$ should be adopted for computing the intensity of double bounce.

Moreover, the radar look angle $\theta$ is the same for all the acquisition time (and so for each subarea in the image) and, obviously, each part of the same wall presents the same orientation $\varphi$ respect to the radar flight trajectory.

As anticipated, in this case the knowledge of the scene geometry is not sufficient in explaying so evident differences in the brightness of the pixels relevant the double reflection.

But Eq.(1) takes into account also the electromagnetic behaviour of the involved materials. Having the wall the same ground in front (mainly grass), there is only one way to explain the effect shown by the SAR image: the east and the west side of the wall present different dielectric constants, i.e. they are made of different materials.

This assumption, even if reasonable according to the scattering model, seemed strange to the authors but has been confirmed by the visit of the site.

In Fig. 5 a picture of the west side of the south wall of the Alte Pinakothek is reported.

The main door corresponds to the centre of the wall. We note the presence of different materials just where it was expected.

In fact, built in 1836, the Alte Pinakothek was severely bombed during the second world war. But the architect Hans Döllgast, engaged in the ' 50 s for the restoration of the gallery, did not reconstruct the building from the beginning.

He used together some old bricks coming from the ruins and new ones and backed the wall with steel pipes because he thought that the wounds of a war have to remain visible to help people to keep the memory alive. Now, looking closely Fig. 2, we can number seven sparkling points. Looking again at Fig. 5, we number seven steel pipes which can be considered the main cause of the different intensity in the double reflection line.

In order to have further confirmation of the theory above, we attempted a new kind of simulation.

A simple geometrical scene has been considered presenting three buildings: in the center, a building with the same dimensions and shape of the Alte Pinakothek has been located with two other buildings nearby.

Some approximations in the geometry have been assumed according to the considerations in the previous Section. For example, windows are not contemplated in the model and the roof, presenting a little slope in the reality, is now flat.

Despite of this geometric simplification, different materials have been considered for the surfaces involved in the double scattering as grass for the ground, bricks for the east side of the wall and steel for the west side.

The result of the SAR image simulation is given in Fig. 6. The image resolution is now $2.57 \mathrm{~m}$ along the azimuth and $4.84 \mathrm{~m}$ in the slant range.
Considering rough a grass soil at the working frequencies of X band [7], GO has been reasonably adopted for computing multiple scattering contributions.

We can clearly see that the brightest side of double reflection where the steel is present is consistent, at least in a qualitative sense, with the analysis performed on real SAR images.

Simulation tools can really confirm the hypothesis, based on the models and also verified visiting the site, of the presence of different materials in the frontal wall of the Alte Pinakoteck.

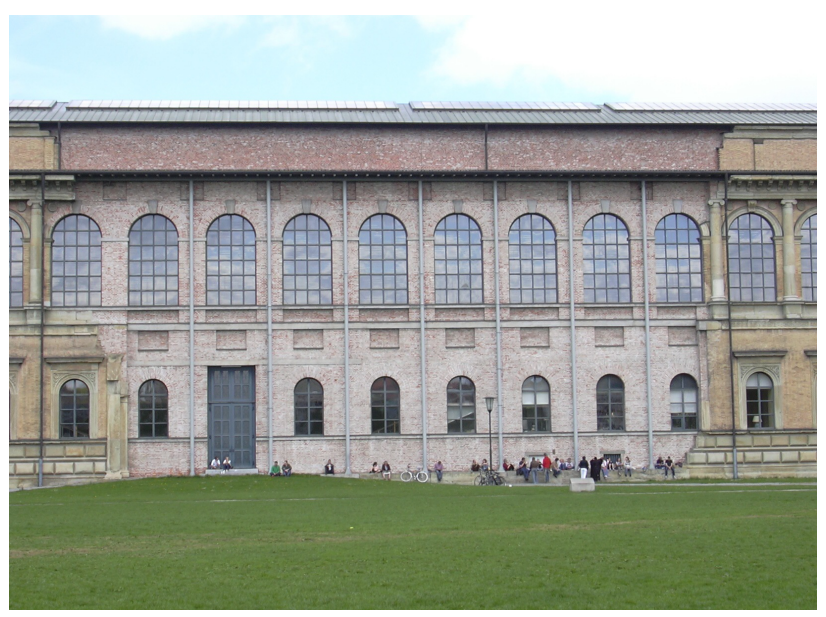

Figure 5. West side of the south wall of the Alte Pinakothek (Munich).

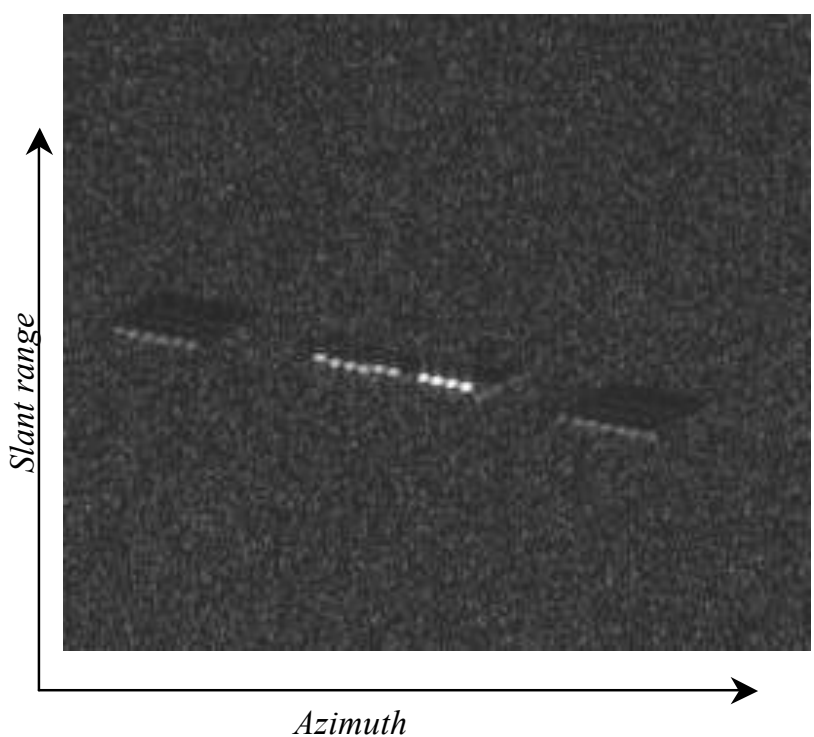

Figure 6. SAR simulated image relevant a canonical scene with two buildings and the Alte Pinakothek. 


\section{CONCLUSIONS}

In this paper, some tools for the study and the analysis of high resolution SAR images, based on a SAR raw signal simulator for urban areas, have been proposed. A real SAR image has been deeply analyzed and different kinds of simulations, considering both the geometric and electromagnetic properties of the scene, have been carried out. Finally, the SAR simulated images have been compared to the real one.

Starting from this comparison, the authors satisfactorily interpret some previously unexplained features of the real SAR image.

In particular, first steps in electromagnetic parameters retrieval have been taken, even if in a qualitative way. Supported by sound scattering models, unexpected difference in building materials has been detected thus assessing the fundamental support of simulation tools to the phase of analysis and understanding of real high resolution SAR data.

\section{REFERENCES}

[1] A.Roth, R.Werninghaus, "Status of the TerraSAR-X Mission", Proceedings of the International Geoscience and Remote Sensing Symposium, Denver (Colorado, USA), pp.1918-1920, 2006.

[2] M.Quartulli, M.Datcu, "Stochastic Geometrical Modeling for Built-Up Area Understanding from a Single SAR Intensity Image with Meter Resolution", IEEE Trans. Geosc. Remote Sensing, vol.42, pp.1996-2003, 2004.

[3] R.Bolter, "Reconstruction of Man-Made Objects from High Resolution SAR Images", IEEE Aerospace Conference Proceedings, vol.3, pp.287292, 2000.

[4] G.Franceschetti, R.Guida, A.Iodice, D.Riccio, G.Ruello, "Deterministic Extraction of Building Parameters from High Resolution SAR Images", Proceedings of the 3rd RSS/ISPRS joint Symposium on Remote Sensing and Data Fusion over Urban Areas, Tempe (Arizona, USA), 2005.

[5] G.Franceschetti, A.Iodice, D.Riccio, G.Ruello "SAR raw signal simulation for urban structures", IEEE Trans. Geosc. Remote Sensing, vol.41, pp.1986-1995, 2003.

[6] G.Franceschetti, A.Iodice, D.Riccio, "A canonical problem in electromagnetic backscattering from buildings", IEEE Trans. Geosc. Remote Sensing, vol.40, pp.1787-1801, 2002.

[7] Z.G.Xia, F.M.Henderson, "Understanding the relationships between radar response patterns and the bio- and geophysical parameters of urban areas", IEEE Trans. Geosc. Remote Sensing, vol.35, pp.93-101, 1997. 